\title{
Parametric Response Mapping of Bronchiolitis Obliterans Syndrome Progression After Lung Transplantation
}

\author{
S. E. Verleden ${ }^{1, *}$, R. $\operatorname{Vos}^{1}$, E. Vandermeulen ${ }^{1}$, \\ D. Ruttens ${ }^{1}$, H. Bellon ${ }^{1}$, T. Heigl ${ }^{1}$, \\ D. E. Van Raemdonck ${ }^{1}$, G. M. Verleden ${ }^{1}$, \\ V. Lama ${ }^{2}$, B. D. Ross ${ }^{3}$, C. J. Galbán ${ }^{3, \dagger}$ and \\ B. M. Vanaudenaerde ${ }^{1, \dagger}$ \\ ${ }^{1}$ Lung Transplant Unit, Department of Clinical and \\ Experimental Medicine, KU Leuven, Leuven, Belgium \\ ${ }^{2}$ Pneumology Department, University of Michigan, Ann \\ Arbor, MI \\ ${ }^{3}$ Radiology Department, University of Michigan, Ann \\ Arbor, MI \\ ${ }^{*}$ Corresponding author: Stijn Verleden, \\ stijn.verleden@med.kuleuven.be \\ 'Both authors contributed equally.
}

Bronchiolitis obliterans syndrome (BOS) remains a major complication after lung transplantation. Air trapping and mosaic attenuation are typical radiological features of BOS; however, quantitative evaluation remains troublesome. We evaluated parametric response mapping (PRM, voxel-to-voxel comparison of inspiratory and expiratory computed tomography [CT] scans) in lung transplant recipients diagnosed with BOS $(n=20)$ and time-matched stable lung transplant recipients $(n=20)$. Serial PRM measurements were performed prediagnosis, at time of BOS diagnosis, and postdiagnosis ( $T_{\text {pre, }}, T_{0}$, and $T_{\text {post }}$, respectively), or at a postoperatively matched time in stable patients. PRM results were correlated with pulmonary function and confirmed by microCT analysis of end-stage explanted lung tissue. Using PRM, we observed an increase in functional small airway disease (fSAD), from $T_{\text {pre }}$ to $T_{0}$ $(p=0.006)$ and a concurrent decrease in healthy parenchyma $(p=0.02)$ in the BOS group. This change in PRM continued to $T_{\text {post }}$, which was significantly different compared to the stable patients $(p=0.0002)$. At BOS diagnosis, the increase in fSAD was strongly associated with a decrease in forced expiratory volume in 1 $s(p=0.011)$. Micro-CT confirmed the presence of airway obliteration in a sample of a BOS patient identified with $67 \%$ fSAD by PRM. We demonstrated the use of PRM as an adequate output to monitor BOS progression in lung transplant recipients.

Abbreviations: AT, air trapping; BOS, bronchiolitis obliterans syndrome; CLAD, chronic lung allograft dysfunction; CT, computed tomography; $\mathrm{FEV}_{1}$, forced expiratory volume in $1 \mathrm{~s}$; fSAD, functional small airway disease; FVC, forced vital capacity; HU, Hounsfield unit; LTx, lung transplantation; PD, parenchymal disease; PRM, parametric response mapping; rCLAD, restrictive CLAD

Received 15 February 2016, revised 07 June 2016 and accepted for publication 12 June 2016

\section{Introduction}

Long-term survival after lung transplantation ( $L T X)$ is hampered by chronic lung allograft dysfunction (CLAD), which is believed to reflect chronic rejection (1). With one of the highest rejection rates among all solid organ transplantations, approximately $50 \%$ of LTx recipients suffer from CLAD within 5 years after transplantation (2). Phenotypes of CLAD with different clinical characteristics and prognoses have been reported in the literature, the most common being bronchiolitis obliterans syndrome (BOS), characterized by an obstructive pulmonary function defect, obliterative bronchiolitis (OB) on histopathologic examination, and air trapping (AT) and mosaic attenuation on imaging. As a result of the complex pathophysiology of these CLAD phenotypes, treating physicians often face difficulties in obtaining an exact and early diagnosis.

The "gold standard" for BOS diagnosis continues to be monitoring of lung function, which is easy to use, costeffective, and provides sufficient repeatability. Lung imaging, primarily via computed tomography (CT), is commonly used to complement spirometry for BOS diagnosis. In BOS, AT is visually identified on end-expiration CT scans as parenchymal areas with low attenuation and lack of volume reduction. De Jong and colleagues proposed a composite CT score for BOS diagnosis, comprising bronchiectasis, mucus plugging, airway wall thickening, consolidation, mosaic pattern, and AT, which was associated with forced expiratory volume in $1 \mathrm{~s}\left(\mathrm{FEV}_{1}\right)$ (3). Although this scoring system showed good reproducibility, this is a semiquantitative technique that requires experienced readers for accurate and repeatable scoring.

Quantitative CT-based analytical measures have shown promise in improving disease diagnosis, phenotypes, and 
prognosis, as well as providing three-dimensional visualization of the disease extent. Parametric response mapping (PRM), a quantitative imaging processing technique applied to inspiration and expiration CT scans, was shown to quantify the extent of parenchyma with emphysematous and nonemphysematous AT, which is referred to as functional small airways disease (fSAD) in a cohort of patients with chronic obstructive pulmonary disease (COPD) (4). In a retrospective study of hematopoietic stem cell recipients, PRM was found to be a strong output of BOS even in the presence of infection (5). Although serial PRM measurements were obtained in only one subject, this technique showed promise in monitoring BOS progression. Here we evaluated PRM as an output of $\mathrm{BOS}$ diagnosis and progression in LTx recipients.

\section{Materials and Methods}

\section{Patient population}

Serial paired CT scans were obtained from LTx recipients diagnosed with BOS $(n=20)$ and recipients without CLAD $(n=20)$ as part of a singlesite retrospective study. Included patients were all double LTx recipients, and both groups were matched for postoperative day of $\mathrm{CT}$, native lung disease, age, and gender. All patients have reached a best postoperative $\mathrm{FEV}_{1}>80 \%$ predicted and all BOS patients received azithromycin treatment for BOS but were found to be nonresponsive. All BOS patients experienced a persistent $\mathrm{FEV}_{1}$ decrease $>20 \%$ compared to the mean of the two best postoperative $\mathrm{FEV}_{1}$ values without a concomitant decrease in total lung capacity ( $T L C) \geq 10 \%$ (thus excluding restrictive CLAD [rCLAD]). Inspiratory and expiratory $C T$ scans from BOS recipients were acquired 3 months to 1 year before BOS, Tpre; at time of BOS diagnosis, $\mathrm{T}_{0}$; and at the last available $\mathrm{CT}, \mathrm{T}_{\text {post, }}$ which may have occurred at end of follow-up, last CT prior to a second LTX, or death. Time-matched CT scans were obtained for the stable recipients. At the time of each CT examination, there was no acute rejection, lymphocytic bronchiolitis, or infection. This study was approved by the local hospital's ethical committee (S57752).

\section{Computed tomography}

CT data were obtained as whole lung volumetric CT scans at full inspiration (TLC) and incremental scans at relaxed expiration (functional residual capacity) on Siemens (Den Haag, The Netherlands) Somatom scanner and reconstructed using a b60 or b70 reconstruction kernel. Slice thicknesses were $1.25 \mathrm{~mm}$ for all scans, with slice numbers on average around 220 for inspiration scans and 15 for expiration scans. All CT scans were checked for Hounsfield unit (HU) drift and if necessary corrected based on aortic blood $(50 \mathrm{HU})$ and central air $(-1000 \mathrm{HU})$ as previously described (6)

\section{Parametric response map}

PRM was applied to all paired CT scans from both study groups. Briefly, lungs from both paired CT scans were segmented from the thoracic cavity using an in-house algorithm written in Matlab (The MathWorks, Inc., Natick, MA). The whole-lung CT inspiration scan was spatially registered and aligned to the incremental CT scan obtained at expiration using Elastix, an open-source image registration algorithm $(7,8)$. Once complete, the images share the same geometric space where each voxel, the smallest unit of volume in a three-dimensional image dataset, consists of $\mathrm{HU}$ values at inspiration and expiration. To minimize the effects of noise on the PRM analysis, an adaptive noise-removal filter was applied to both spatially aligned CT scans. Individual voxels were then classified based on predetermined thresholds as normal (green), fSAD (yellow), emphysema (red), and parenchymal disease (purple). Voxels with values $\geq-950 \mathrm{HU}$ and $<-810 \mathrm{HU}$ at inspiration and $\geq-856 \mathrm{HU}$ at expiration were classified normal, $\geq-950 \mathrm{HU}$ and $<-810 \mathrm{HU}$ at inspiration and $<-856$ at expiration were fSAD, $<-950 \mathrm{HU}$ at inspiration and $<-856 \mathrm{HU}$ at expiration were emphysema, and $\geq-810 \mathrm{HU}$ at inspiration were parenchymal disease (PD) (5). The emphysema and AT thresholds ( $-950 \mathrm{HU}$ and $-856 \mathrm{HU}$, respectively) have been previously defined and are used for CT analysis in the COPDGene clinical trial (9-12). The upper limit on the inspiratory CT $(-810 \mathrm{HU})$ was determined using healthy smokers accrued as part of the NORM trial $(5,13)$. Global PRM measures were presented as relative lung volumes. The relative volumes for each classification are defined as the sum of all voxels within a class normalized to the sum of all voxels within the volume of the expiratory lungs multiplied by 100 . PRM Normal, PRM $^{\text {fSAD }}$, PRM $^{E m p h}$, and PRM ${ }^{\text {PD }}$ denote the relative volume for each classification.

\section{MicroCT investigation}

Further evaluation of the PRM technique was performed by microCT analysis of an explanted lung from a single recipient that had undergone whole-lung inspiration and expiration CT scans immediately prior to a redo LTX. The lung specimen was collected and scanned using an adapted protocol of our previous work $(14,15)$ (online supplement 1, Figure S1).

\section{Statistical analysis}

All results are shown as median (interquartile range). Differences in continuous variables between BOS and stable patients were determined using Student's t-test. A contingency test was used to assess group difference in discrete data. Paired t-test was used to assess differences between time points (i.e. $T_{\text {pre, }}, T_{0}$, and $T_{\text {post }}$ ). In addition to the absolute values determined by PRM, the relative change in PRM ${ }^{\text {Normal }}$ and PRM ${ }^{\mathrm{FSAD}}$ were evaluated between time points and groups. The relative change in PRM metric is defined as $100 *\left[\operatorname{PRM}^{i}\left(\mathrm{~T}_{\mathrm{j}}\right)-\operatorname{PRM}^{i}\left(\mathrm{~T}_{\text {pre }}\right)\right] / \operatorname{PRM}^{i}\left(\mathrm{~T}_{\text {pre }}\right)$, where $i$ is an index to denote normal and fSAD and $j$ denotes time points $\left(T_{0}, T_{\text {post }}\right)$. Spearman-rank test was performed for correlation analysis between the relative change in pulmonary function measurements and PRM ${ }^{\mathrm{FAD}}$. Receiver operating characteristic curve $(\mathrm{ROC})$ analysis was performed to estimate the specificity and sensitivity of $\mathrm{PRRM}^{\dagger S A D}$ at $T_{0}$ to detect BOS using the entire study population $(n=40)$. All tests were performed using graph pad prism 6.0 (GraphPad Software, Inc., La Jolla, CA).

\section{Results}

\section{Study population}

Patient characteristics are presented in Table 1. Patients were selected to minimize potentially confounding effects as well as to time-match postoperative CT acquisitions. As such, no significant differences in age, underlying disease, gender, and postoperative day of CT scanning between the BOS and stable groups were observed. At $T_{\text {pre, }}$ nine patients from the BOS group had BOSOp versus five in the stable group. Four patients in the stable group had BOSOp at $T_{0}$, which increased to nine patients at $\mathrm{T}_{\text {post }}$. However, none of the stable patients reached BOS1 at any time point.

\section{Serial examination of PRM measurements}

Representative PRM slices from two cases are shown in Figure 1. Population results for serial $P R M^{f S A D}$ and 
Table 1: Patient characteristics of the 20 BOS and 20 stable patients included in the PRM study

\begin{tabular}{|c|c|c|c|}
\hline & BOS & Stable & p-value \\
\hline Number of patients, $n$ & 20 & 20 & \\
\hline Donor smoking, n (\%) & & & 0.56 \\
\hline Yes & $3(15 \%)$ & $5(25 \%)$ & \\
\hline No & $13(65 \%)$ & $13(65 \%)$ & \\
\hline Unknown & $4(20 \%)$ & $2(10 \%)$ & \\
\hline Donor age, years & $42 \pm 4$ & $43 \pm 3$ & 0.93 \\
\hline Recipient age, years & $46 \pm 3$ & $49 \pm 3$ & 0.54 \\
\hline Recipient gender, n (\%) & & & 0.75 \\
\hline Male & $8(40 \%)$ & $9(45 \%)$ & \\
\hline Female & $12(60 \%)$ & $11(55 \%)$ & \\
\hline Underlying disease, $\mathrm{n}(\%)$ & & & 0.75 \\
\hline COPD & $9(45 \%)$ & $11(55 \%)$ & \\
\hline $\mathrm{CF}+\mathrm{BRECT}$ & $3(15 \%)$ & $5(25 \%)$ & \\
\hline ILD & $2(10 \%)$ & $1(5 \%)$ & \\
\hline Redo & $4(20 \%)$ & $2(10 \%)$ & \\
\hline Eisenmenger & $1(5 \%)$ & $1(5 \%)$ & \\
\hline Histiocytosis X & $1(5 \%)$ & 0 & \\
\hline \multicolumn{4}{|l|}{ POD of CT, years } \\
\hline Time point $1, T_{\text {pre }}$ & $2.7 \pm 0.4$ & $2.6 \pm 0.4$ & 0.90 \\
\hline Time point $2, T_{0}$ & $3.3 \pm 0.5$ & $3.5 \pm 0.5$ & 0.80 \\
\hline Time point $3, T_{\text {post }}$ & $4.9 \pm 0.4$ & $5.0 \pm 0.6$ & 0.82 \\
\hline \multicolumn{4}{|l|}{$\mathrm{FEV}_{1}$ at $\mathrm{CT}, \mathrm{L}$} \\
\hline Time point $1, T_{\text {pre }}$ & $2.65 \pm 0.16$ & $3.01 \pm 0.17$ & 0.14 \\
\hline Time point $2, T_{0}$ & $1.88 \pm 0.15$ & $3.12 \pm 0.17$ & $<0.0001$ \\
\hline Time point $3, T_{\text {post }}$ & $1.11 \pm 0.15$ & $3.02 \pm 0.19$ & $<0.0001$ \\
\hline \multicolumn{4}{|l|}{ FEF25-75 at CT, L } \\
\hline Time point $1, T_{\text {pre }}$ & $2.54 \pm 0.26$ & $3.05 \pm 0.29$ & 0.19 \\
\hline Time point $2, \mathrm{~T}_{0}$ & $1.17 \pm 0.24$ & $2.97 \pm 0.29$ & $<0.0001$ \\
\hline Time point $3, T_{\text {post }}$ & $0.41 \pm 0.07$ & $2.79 \pm 0.24$ & $<0.0001$ \\
\hline \multicolumn{4}{|c|}{ BOS stage at diagnosis, n (\%) } \\
\hline BOS1 & $13(65 \%)$ & NA & \\
\hline BOS2 & $4(20 \%)$ & NA & \\
\hline BOS3 & $3(15 \%)$ & NA & \\
\hline
\end{tabular}

Contingency tables were used to compare group differences, unpaired t-test was used for continuous data.

BOS, bronchiolitis obliterans syndrome; BRECT, bronchiectasis; CF, cystic fibrosis; COPD, chronic obstructive pulmonary disease; CT, computed tomography; FEF, forced expiratory flow rate; ILD, interstitial lung disease; NA, not applicable; POD, postoperative day; PRM, parametric response mapping; Redo, redo lung transplantation.

$\mathrm{PRM}^{\text {Normal }}$ measurements are shown in Figures 2A and B. BOS patients demonstrated a significant increase in PRM $^{\text {fSAD from }} \mathrm{T}_{\text {pre }}(18 \pm 3 \%)$ to $\mathrm{T}_{0} \quad(33 \pm 4 \%$; $\mathrm{p}=0.006)$. Although the moderate increase in $P R M^{\mathrm{fSAD}}$ from $T_{0}$ to $T_{\text {post }}(45 \pm 6 \%)$ was not significant ( $\left.p=0.18\right)$, $\mathrm{PRM}^{\text {fSAD }}$ at $\mathrm{T}_{\text {post }}$ was found to be significantly higher compared to $T_{\text {pre }}$ values ( $p<0.0001$ ). In stable patients, a negligible variation in $\mathrm{PRM}^{\mathrm{fSAD}}$ was observed from $T_{\text {pre }}$ to $T_{0}$ and $T_{\text {post }}(p=0.27$ and $p=0.28$, respectively). PRM $^{\text {Normal }}$ decreased from $T_{\text {pre }}$ to $T_{0}(62 \pm 4 \%$ to $48 \pm 4 \%, p=0.02)$ in BOS patients with no further decline after diagnosis, $T_{\text {post }}(38 \pm 6 \%, p=0.36)$. However, $P R M^{\text {Normal }}$ at $T_{\text {post }}$ demonstrated a significant decrease from $T_{\text {pre }}$ values $(p=0.0007)$. Negligible changes were observed in $\mathrm{PRM}^{\text {Normal }}$ for the control group $\left(53 \pm 4 \%\right.$ at $\mathrm{T}_{\text {pre }}, 61 \pm 5 \%$ at $\mathrm{T}_{0}$, and $55 \pm 6 \%$ at $\left.\mathrm{T}_{\text {post }}\right)$.

\section{Group comparisons}

Negligible group differences were observed in PRM values of fSAD and healthy parenchyma $(p=0.17$ and $p=0.14$, respectively; Figures $2 A$ and $B$ ) and $F E V_{1}$

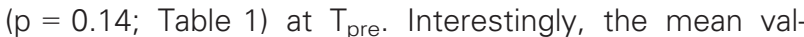
ues for $P R M^{f S A D}$ at $T_{\text {pre }}$ were slightly higher in both groups (BOS and stable had $18 \pm 3 \%$ and $24 \pm 4 \%$, respectively) compared to a previously described healthy (nontransplant) control group $(<10 \%$ [4] and $<15 \%$, supplemental material [5]). Although $\mathrm{PRM}^{\mathrm{fSAD}}$ was higher at $T_{0}$ for BOS patients than stable patients, these values were not significant $(p=0.09$; Figure 2A). The steady increase in $\mathrm{PRM}^{\mathrm{fSAD}}$ in BOS patients resulted in a significant difference between BOS and stable patients at $T_{\text {post }}(p=0.0002)$. Similarly, $P R M^{\text {Normal }}$ were significantly lower at $T_{0}(p=0.02)$ and $T_{\text {post }}(p=0.04)$ in BOS patients when compared to stable patients (Figure 2B). To evaluate whether PRM ${ }^{\mathrm{fSAD}}$ can detect BOS severity, patients diagnosed with BOS were separated based on BOS grade. Results from this analysis found that patients with BOS 2-3 $(n=7)$ at diagnosis had three times more PRM ${ }^{\text {SAD }}(26 \pm 7 \%)$ than BOS 1 patients $(n=13 ; 9 \pm 7 \%)$. Although this result was not found to be significant $(p=0.10)$, the general trend suggests $\mathrm{PRM}^{\mathrm{fSAD}}$ may provide clinical insight into BOS severity. At the end of current follow-up ( $\left.T_{\text {post }}\right), 11$ patients had graft loss (4 deaths, 7 redo LTX) with 9 patients alive (BOS1, $n=4$; BOS2, $n=4$, BOS3, $n=1$ ) compared to no graft loss in the stable group. Patients who succumbed to BOS (all BOS 3) had elevated $\mathrm{PRM}^{\mathrm{fSAD}}(59 \pm 2 \%)$ compared to living patients $(25 \pm 6 \%) \quad(p=0.0007)$, while PRM $^{\text {Normal }}$ was significantly lower $(21 \pm 3 \% \quad$ vs. $56 \pm 8 \%$ respectively, $p=0.0007)$.

\section{Relative change in PRM}

The relative change in PRM ${ }^{\mathrm{fSAD}}\left(\mathrm{rPRM}^{\mathrm{fSAD}}\right.$ ) and $\mathrm{PRM}^{\text {Normal }}$ ( $r \mathrm{PRM}^{\text {Normal}}$ ) from $\mathrm{T}_{\text {pre }}$ are presented in Figures $2 \mathrm{C}$ and $\mathrm{D}$. As shown in Figure 2C, patients diagnosed with BOS were found to have a relative increase in $\mathrm{PRM}^{\mathrm{fSAD}}$ of $422 \pm 127 \%$ at $\mathrm{T}_{0} \quad(\mathrm{p}=0.005)$ and $436 \pm 95 \%$ at $T_{\text {post }}$. These values were significantly higher than rPRM $^{\mathrm{fSAD}}$ observed in stable patients $\left(\mathrm{rPRM}^{\mathrm{fSAD}}\right.$ at $\mathrm{T}_{0}$ of $92 \pm 16 \% \quad(p=0.004)$ and at $\mathrm{T}_{\text {post }}$ $116 \pm 30 \% \quad(p=0.001)$. As presented in Figure 2D, rPRM $^{\text {Normal }}$ was found to be lower in BOS patients as compared to stable patients. These values were significantly different between groups at $T_{0}(p=0.004)$, but lost significance by $T_{\text {post }}(p=0.07)$. The extent of change in rPRM $^{\text {Normal }}$ was only significant at $T_{\text {post }}$ for BOS patients $(p=0.009)$. By ROC analysis, the relative increase in $\mathrm{PRM}^{\mathrm{fSAD}}$ at $\mathrm{T}_{0}$ was found to be significantly associated with BOS ( $p=0.005)$. An optimal cutoff of $r P R M^{f S A D}$ at $T_{0}$ was found to be $173 \%$, which had $62.5 \%$ sensitivity and 93.8\% specificity to predict BOS development. 

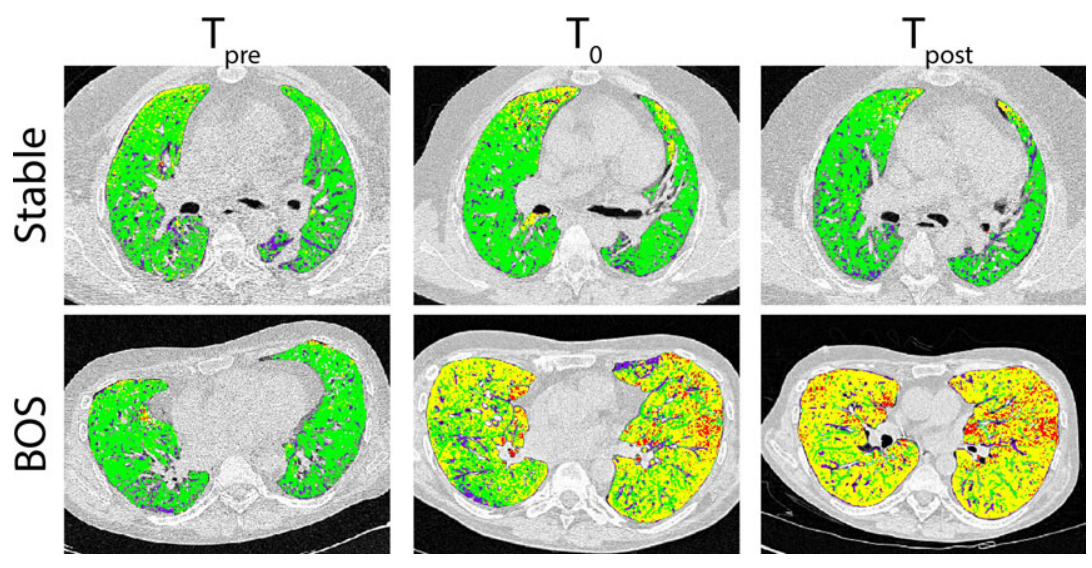

Figure 1: Presented are representative axial slices of PRM from two cases demonstrating the evolution of fSAD in a stable patient (upper panel) and a BOS patient (lower panel) at $\mathbf{T}_{\text {pre, }} \mathbf{T}_{\mathbf{0}}$, or $\mathbf{T}_{\text {post }}$ (left to right). The stable case is a male, 40 years of age, who underwent LTx for COPD. The PRM values at 5 years posttransplantation $\left(T_{\text {pre }}\right)$ for normal and fSAD were $71 \%$ and $12 \%$ of the lung volume, respectively. Negligible changes in these PRM values were observed 5.5 years $\left(T_{0} ; P^{2} M^{\text {Normal }}=77 \%\right.$ and $\left.\mathrm{PRM}^{\mathrm{fSAD}}=11 \%\right)$ and 7 years $\left(\mathrm{T}_{\text {post }} \mathrm{PRM}^{\mathrm{Normal}}=78 \%\right.$ and $\left.\mathrm{PRM}^{\mathrm{fSAD}}=6 \%\right)$ posttransplantation. The BOS case is a $36-\mathrm{year}-\mathrm{old}$ male transplanted for histocytosis X. PRM ${ }^{\text {Normal }}$ and PRM ${ }^{\text {fSAD }}$ at 5 years posttransplantation were $76 \%$ and $13 \%$, respectively. This patient was diagnosed with BOS 5.5 years posttransplantation $\left(T_{0}\right)$. At $T_{0}, P_{R} M^{\text {Normal }}$ was $30 \%$ of the lung volume with $P R M^{f S A D}$ accounting for $56 \%$. Prior to redo-transplantation (6 years posttransplantation), PRM $^{\text {fSAD }}$ changed slightly from the $T_{0}$ value $(58 \%)$, yet PRM ${ }^{\text {Normal }}$ decreased to $18 \%$ of the lung volume. The resulting loss of PRM ${ }^{\text {Normal }}$ is attributed to an increase of low-attenuation regions identified by PRM as emphysema ( $1 \%$ at $\mathrm{T}_{0}$ to $12 \%$ at $\mathrm{T}_{\text {post }}$ ). BOS, bronchiolitis obliterans syndrome; COPD, chronic obstructive pulmonary disease; fSAD, functional small airway disease; LTX, lung transplantation; PRM, parametric response mapping.

\section{Correlation of PRM ${ }^{\dagger S A D}$ with spirometric measures and $A T$}

Correlations were performed in BOS patients to determine the relationship between PRM and pulmonary function (Figure 3). A relative decline in $\mathrm{FEV}_{1}$ (\% pred) from $T_{\text {pre to }} T_{0}$ was inversely associated with PRM ${ }^{f S A D}$ $(p=0.006$ and $R=-0.64)$. Similarly, the decline in forced expiratory flow rate 25-75 was inversely correlated with $\operatorname{PRM}^{\mathrm{fSAD}}(\mathrm{p}=0.031$ and $\mathrm{R}=-0.53)$. In contrast, no association was observed for the relative change in forced vital capacity (FVC) (\%pred) ( $p=0.43$ ). The inverse correlation between $\mathrm{PRM}^{\mathrm{fSAD}}$ to both relative changes in $\mathrm{FEV}_{1}$ (\%pred) and FVC (\%pred) became stronger from $T_{\text {pre }}$ to $T_{\text {post }}(p=0.0002, R=-0.59$ and $p=0.0006$ and $R=-0.53$, respectively). A correlation analysis using the absolute value of $\mathrm{FEV}_{1}$ and FVC in liters yielded similar results (data not shown).

\section{Confirmation by microcT}

Presented in Figure 4 are PRM and microCT results from a representative BOS explant specimen. Using CT scans acquired at $T_{\text {post, }}$ this patient was found to have PRM measures over both lungs of $15 \%$ for healthy parenchyma, $56 \%$ for $f S A D, 19 \%$ for $P D$, and $4 \%$ for emphysema. As seen in Figures $4 A$ and $B$, the PRM, with corresponding scatter plot, at the exact location of the selected core showed a high percentage of fSAD $\left(\mathrm{PRM}^{\mathrm{fSAD}}=67 \%\right)$ with lower values in PD $\left(\mathrm{PRM}^{\mathrm{PD}}=16 \%\right)$, normal parenchyma $\left(\mathrm{PRM}^{\text {Normal }}=9 \%\right)$, and emphysema $\left(\mathrm{PRM}_{\mathrm{Emph}}=1 \%\right)$. MicroCT confirmed the presence of small airway obliteration on two separate locations within the single scanned specimen (Figures $4 \mathrm{C}$ and $\mathrm{D}$ ), which is compatible with $\mathrm{OB}$, the pathological correlate of BOS.

\section{Discussion}

In this article, we demonstrated the potential value of serial radiological PRM measurements in diagnosing and monitoring BOS. We provided further support for our findings by demonstrating a correlation between $\mathrm{PRM}^{\text {fSAD }}$ and FEV ${ }_{1}$. Lastly, microCT examination confirmed the presence of small airway obliteration, explaining the FSAD documented with the PRM measurement.

An interesting find in this study was the elevated $\mathrm{PRM}^{\mathrm{fSAD}}$ observed in all $L T x$ recipients prior to any pulmonary function defect. These values were higher than observed from nontransplant healthy smokers reported in the literature $(4,5)$. Although all patients in this study had a $\mathrm{FEV}_{1}>80 \%$ predicted at $\mathrm{T}_{\text {pre, }}$ this find corroborates the observation that AT occurs early after LTx, even in stable patients. This is thought to be attributed to some degree of small airway disease secondary to the transplantation procedure (16). Also, more extended criteria donors, typically older with a longer smoking history, are more commonly used (17).

Recently, Solyanik et al compared three methods of quantifying AT in LTx patients (18). Their approach differed from the present study in that multiple 
A

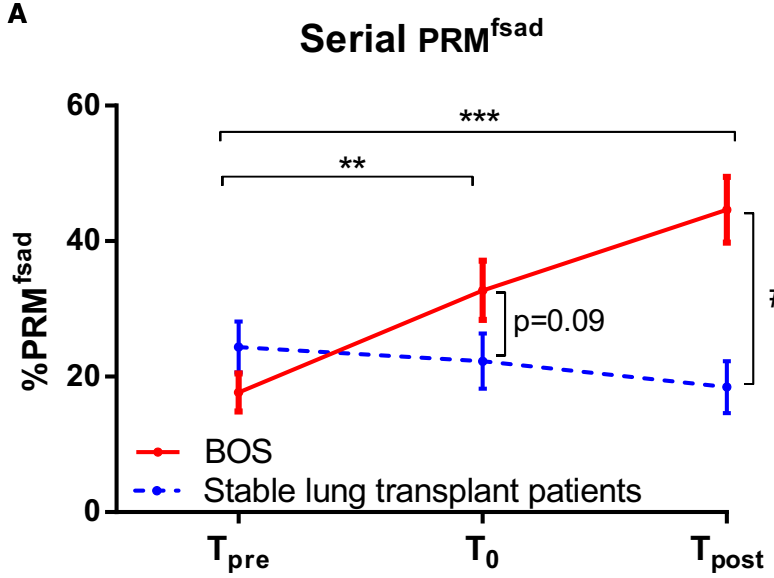

B

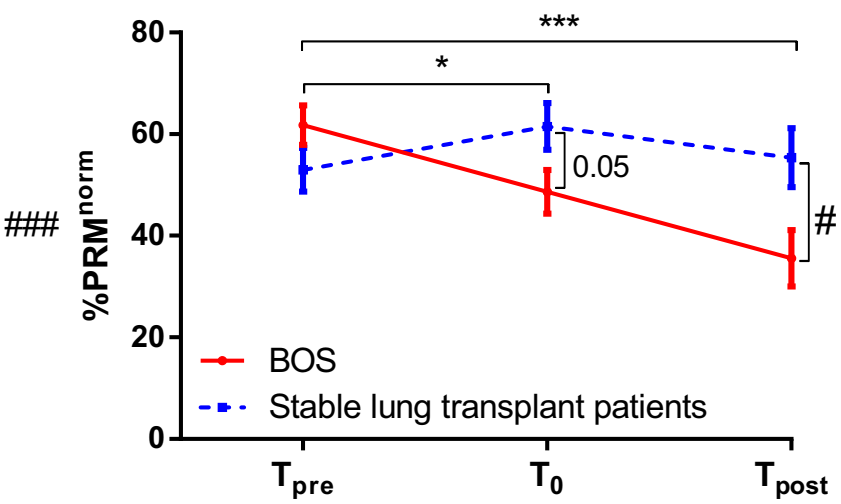

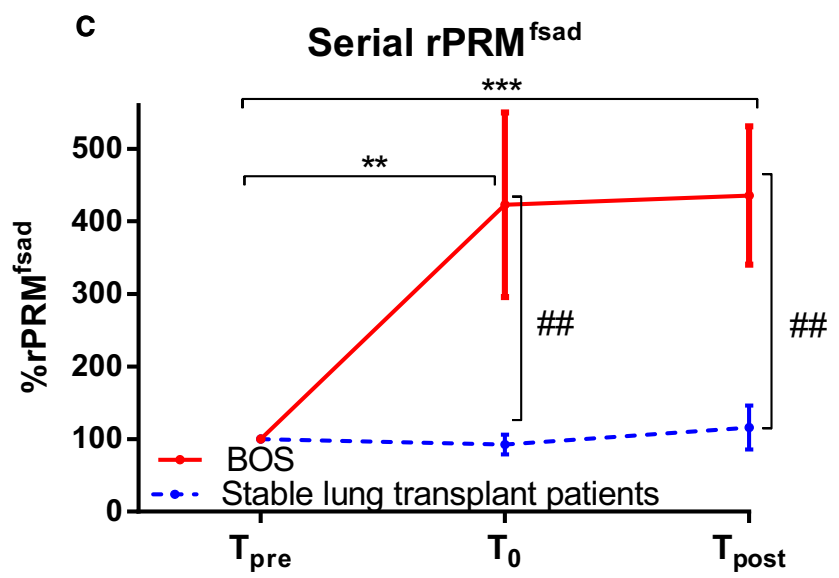

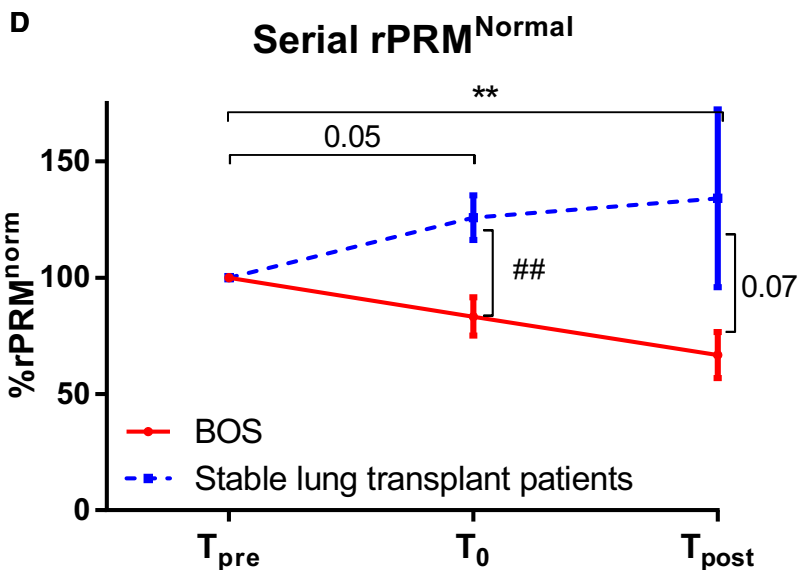

Figure 2: Group comparisons of serial measurements of PRM. Line plots are used to present the group differences observed for the absolute and relative change in (A and C) PRM ${ }^{f S A D}$ and (B and D) PRM ${ }^{\text {Normal }}$ obtained at $T_{\text {pre, }} T_{0}$, and $T_{\text {post }}$. Significant differences between time points for the BOS groups is denoted as ${ }^{*}$ for $p<0.05,{ }^{*}$ for $p<0.01$, and ${ }^{* * *}$ for $p<0.001$. Significant dif-

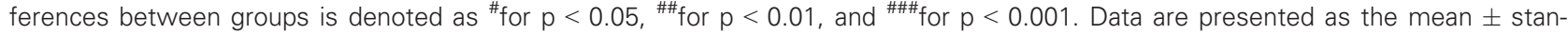
dard error of the mean. BOS, bronchiolitis obliterans syndrome; PRM, parametric response mapping.

measurements within the same patients were not obtained. Nevertheless, one method applied a density mapping approach with voxel-to-voxel comparison of inspiratory and expiratory CT analogous to PRM, with the difference being the use of a single threshold applied to the expiratory CT scan. The authors demonstrated a correlation with residual volume/total lung capacity (used to define pathologic AT) and the density mapping technique. In parallel, we also quantified AT by radiological scoring and compared this to PRM (online supplement 2; Figure S2). Although AT progressively increased in BOS patients, we were unable to demonstrate a correlation between $\mathrm{FEV}_{1}$ decrease and increase in AT, demonstrating that PRM might be a more sensitive and objective measurement for estimating small airway obstruction compared to subjective scoring, as PRM is probably better in detecting subtle differences in AT.
Interestingly, in end-stage BOS lungs, 59\% of the lung was found to be fSAD by PRM. This finding corresponds well with the previously observed percentage of obstructed airways we calculated using our previously reported ex-vivo CT analysis of explanted lungs, where we found that between $50 \%$ and $60 \%$ of the airways were obliterated from generation 7 on (14). PRM ${ }^{\text {fSAD }}$ generated from the core $(67 \%)$ slightly differed from what was calculated over both lungs (56\%). This is most likely attributed to spatial heterogeneity of the disease within the lungs. Therefore, severe airway obstruction observed in the analyzed lung strengthens the utility of PRM as a viable tool for monitoring BOS progression.

The objective of this study was to evaluate PRM as an indicator of BOS progression. As such, patients with rCLAD were deliberately excluded as they demonstrate 
A

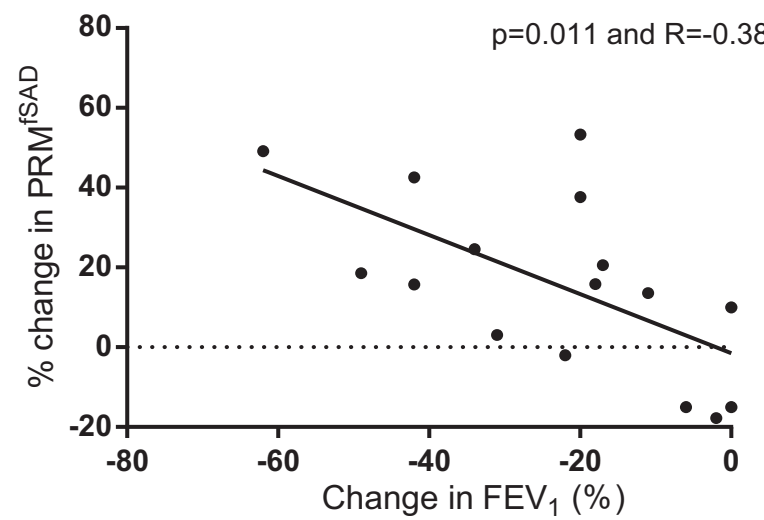

B

$\mathrm{T}_{\text {pre }}-\mathrm{T}_{\text {post }}$

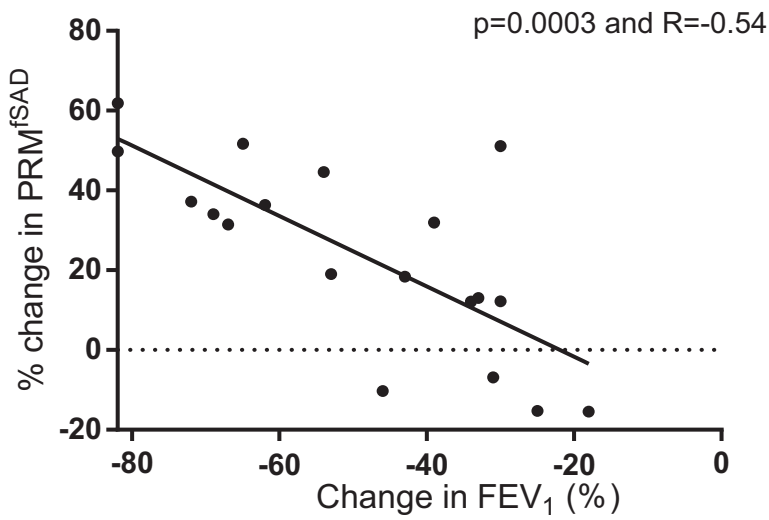

Figure 3: Assessment of the relationship between PRM and pulmonary function. Correlation analyses are presented for the relative change in $P R M^{f S A D}$ to the relative change in $F E V_{1}$ from $(A) T_{\text {pre }}$ to $T_{0}$ and $(B) T_{\text {pre }}$ to $T_{\text {post. }} . F E V_{1}$, forced expiratory volume in $1 \mathrm{~s}$; $f S A D$, functional small airway disease; PRM, parametric response mapping.
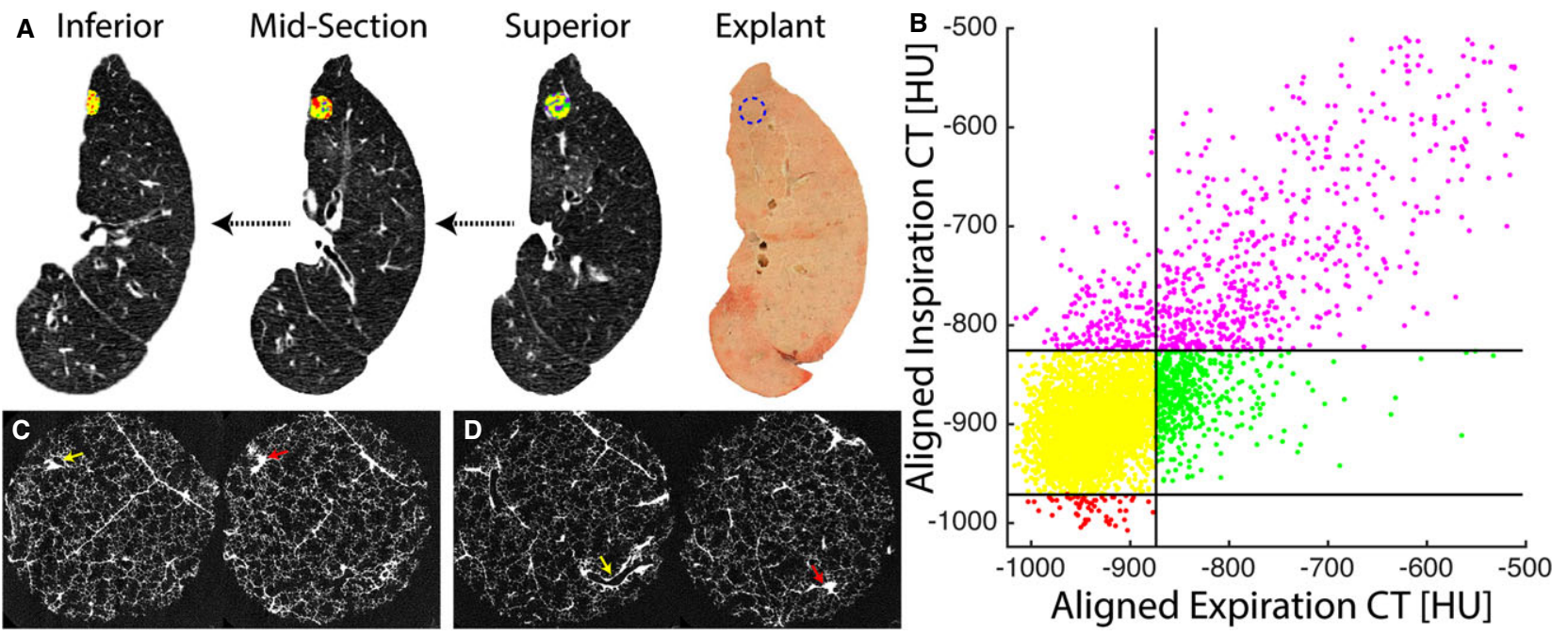

Figure 4: Confirmation of $\mathrm{OB}$ by microCT in a lung region of high $\mathbf{P R M}^{\mathbf{f S A D}}$. (A) Presented are serial in vivo expiration CT Scans with PRM image or cored region from superior to inferior and the explant slice with the location of the scanned core indicated by a blue dashed circle. (B) Corresponding PRM scatter plot for the cored region. The PRM at the exact location of the selected core showed a high percentage of $f S A D$, yellow $\left(P R M^{f S A D}=67 \%\right)$; while high attenuation regions, purple $\left(P R M{ }^{P D}=16 \%\right)$ and normal parenchyma, green $\left(\mathrm{PRM}^{\mathrm{Norm}}=9 \%\right)$ were less frequent, while emphysema was almost absent, red $(\mathrm{PRM} \mathrm{Emph}=1 \%)$. (C) MicroCT images of the core showing initially a patent airway (yellow arrow), which completely obliterates (red arrow). (D) MicroCT images within the same core at a different location illustrating another airway lesion where a patent airway (yellow arrow) is found to completely obliterate (red arrow). CT, computed tomography; fSAD, functional small airway disease; HU, Hounsfield unit; OB, obliterative bronchiolitis; PRM, parametric response mapping.

elevated attenuation on inspiration CT scans as a result of central and peripheral ground glass opacities (19-21). Since PRM has not been validated yet in restrictive lung disease, these patients were not further investigated.

Other possible limitations include the relatively low number of patients included in this study, which complicates analyses that require further group stratification. PRM is a quantitative density-based CT technique that applies thresholds for sorting individual voxels into specific classifications. As such, various sources of variability may alter the density measurements affecting PRM classifications. In our previous work, we evaluated the effect these sources have on altering the PRM measurements (22). The limitations and generalizability of PRM are further discussed in online supplement 3 . However, all CT scans in 


\section{Verleden et al}

this study design were quality controlled by assessing $\mathrm{HU}$ drift and corrected when needed. In an effort to reduce the effects of variation in CT acquisitions and reconstruction kernels, a noise-reducing filter was applied to all scans prior to PRM analysis. It is possible that the PRM thresholds used may not fully represent the disease state of the studied patient population. PRM ${ }^{\mathrm{fSAD}}$ was found to be elevated in pre-BOS recipients relative to healthy smokers, which was consistent with observations from a previous study (5). This may be a consequence of the expiratory threshold (i.e. $-856 \mathrm{HU}$ ) overestimating the extent of AT in the lungs. Confirmation of PRM ${ }^{f S A D}$ by microCT analysis of explanted lungs alleviates some of these possible concerns as OB lesions were observed in regions with high $\mathrm{PRM}^{\mathrm{fSAD}}$. The big advantage of our study is the serial measurements from a stable to a diseased state (BOS), which has, to the best of our knowledge, not been performed in this context. It is well understood that spirometric decline is a hallmark of the onset of BOS. Yet clinical practice dictates that CT scans must be used to rule out other pulmonary complications that cause a decline in pulmonary function and to potentially differentiate with rCLAD. As CT scans are part of standard clinical management of these patients, PRM analysis of these scans may complement $\mathrm{FEV}_{1}$ as an objective output of the presence and extent of $\mathrm{OB}$ as indicated by PRM ${ }^{\text {fSAD }}$ and might be specifically useful in patients in whom spirometry is difficult to interpret such as suture problems, pneumonectomy, or single lung transplanted patients (see online supplement 4 and Figure S3 for a representative example of a patient with fluctuating $\mathrm{FEV}_{1}$ but stable PRM ${ }^{\mathrm{fSAD}}$ values).

In conclusion, we demonstrate that PRM served as an imaging output of BOS. Further proof of its applicability is provided by the correlation with spirometry and the presence of $\mathrm{OB}$ with microCT. PRM investigation may serve as a complementary tool to aid treating physicians in diagnosing BOS. Further research in this field would include a large, multicenter prospective trial and inclusion of patients with rCLAD. These steps could further confirm the potential use and benefit of PRM for monitoring CLAD progression in LTX recipients.

\section{Acknowledgments}

Part of this study was supported by the US National Institutes of Health Research grants R01HL122438 and R44HL11837, S.E.V. and R.V. are sponsored by FWO (12G8715N and $1803516 \mathrm{~N})$. R.V. is supported by the Research Foundation Flanders (FWO) (KAN2014 1.5.139.14) and starting grant UZ Leuven (STG/15/023). G.M.V. and B.M.V. are supported by the FWO (G.0723.10 and G.0679.12) and KU Leuven Research Funding C24/ $15 / 030$.

\section{Disclosure}

The authors of this manuscript have conflicts of interest to disclose as described by the American Journal of
Transplantation. C.J.G. and B.D.R. have a financial interest in the underlying patented University of Michigan technology licensed to Imbio, LLC., a company in which B.D.R. has a financial interest. The other authors have no conflicts of interest to disclose.

\section{References}

1. Verleden GM, Raghu G, Meyer KC, Glanville AR, Corris P. A new classification system for chronic lung allograft dysfunction. J Heart Lung Transplant 2014; 33: 127-133.

2. Yusen RD, Edwards LB, Kucheryavaya AY, et al. The Registry of the International Society for Heart and Lung Transplantation: Thirty-second Official Adult Lung and Heart-Lung Transplantation Report-2015; Focus Theme: Early Graft Failure. J Heart Lung Transplant 2015; 34: 1264-1277.

3. De Jong PA, Dodd JD, Coxson HO, et al. Bronchiolitis obliterans following lung transplantation: early detection using computed tomographic scanning. Thorax 2006; 61: 799-804.

4. Galbán CJ, Han MK, Boes JL, et al. Computed tomographybased biomarker provides unique signature for diagnosis of COPD phenotypes and disease progression. Nat Med 2012; 18 : 1711-1715.

5. Galbán CJ, Boes JL, Bule M, et al. Parametric response mapping as an indicator of bronchiolitis obliterans syndrome after hematopoietic stem cell transplantation. Biol Blood Marrow Transplant 2014; 20: 1592-1598.

6. Stoel BC, Stolk J. Optimization and standardization of lung densitometry in the assessment of pulmonary emphysema. Invest Radiol 2004; 39: 681-688.

7. Klein S, Staring M, Murphy K, Viergever MA, Pluim JPW. Elastix: A toolbox for intensity-based medical image registration. IEEE Trans Med Imaging 2010; 29: 196-205.

8. Shamonin DP, Bron EE, Lelieveldt BPF, et al. Fast parallel image registration on $\mathrm{CPU}$ and GPU for diagnostic classification of Alzheimer's disease. Front Neuroinform 2013; 7: 50.

9. Regan EA, Hokanson JE, Murphy JR, et al. Genetic epidemiology of COPD (COPDGene) study design. COPD 2010; 7: 32-43.

10. Gevenois PA, de Maertelaer V, De Vuyst P, Zanen J, Yernault JC. Comparison of computed density and macroscopic morphometry in pulmonary emphysema. Am J Respir Crit Care Med 1995; 152: 653-657.

11. Gevenois PA, De Vuyst P, de Maertelaer V, et al. Comparison of computed density and microscopic morphometry in pulmonary emphysema. Am J Respir Crit Care Med 1996; 154: 187-192.

12. Newman KB, Lynch DA, Newman LS, Ellegood D, Newell JD. Quantitative computed tomography detects air trapping due to asthma. Chest 1994; 106: 105-109.

13. Boudewijn IM, Postma DS, Telenga ED, et al. Effects of ageing and smoking on pulmonary computed tomography scans using parametric response mapping. Eur Respir J 2015; 46: 11931196.

14. Verleden SE, Vasilescu DM, Willems S, et al. The site and nature of airway obstruction after lung transplantation. Am J Respir Crit Care Med 2014; 189: 292-300.

15. Verleden SE, Vasilescu DM, McDonough JE, et al. Linking clinical phenotypes of chronic lung allograft dysfunction to changes in lung structure. Eur Respir J 2015; 46: 1430-1439.

16. Bankier AA, Van Muylem A, Knoop C, Estenne M, Gevenois PA. Bronchiolitis obliterans syndrome in heart-lung transplant recipients: Diagnosis with expiratory CT. Radiology 2001; 218: 533539. 
17. Somers J, Ruttens D, Verleden SE, et al. A decade of extendedcriteria lung donors in a single center: Was it justified? Transpl Int 2015; 28: 170-179.

18. Solyanik O, Hollmann P, Dettmer S, et al. Quantification of pathologic air trapping in lung transplant patients using CT density mapping: Comparison with other CT air trapping measures. PLoS One 2015; 10: e0139102.

19. Verleden SE, de Jong PA, Ruttens D, et al. Functional and computed tomographic evolution and survival of restrictive allograft syndrome after lung transplantation. J Heart Lung Transplant 2014; 33: 270-277.

20. Sato M, Waddell TK, Wagnetz $U$, et al. Restrictive allograft syndrome (RAS): A novel form of chronic lung allograft dysfunction. J Heart Lung Transplant 2011; 30: 735-742.

21. Todd JL, Jain R, Pavlisko EN, et al. Impact of forced vital capacity loss on survival after the onset of chronic lung allograft dysfunction. Am J Respir Crit Care Med 2014; 189: 159-166.

22. Boes JL, Bule M, Hoff BA, et al. The impact of sources of variability on parametric response mapping of lung CT scans. Tomography 2015; 1: 69-77.

\section{Supporting Information}

Additional Supporting Information may be found in the online version of this article.
Data S1: Supplemental materials and methods.

Figure S1: (A) deformable registration of the in vivo inspiration, in vivo expiration, and explant lung CT. (B) Linear registration of the explant $\mathrm{CT}$ with the specific (drilled) lung section. (C) Final transformation of the in vivo and expiration $\mathrm{CT}$.

Figure S2: Comparison of $\mathrm{PRM}_{\mathrm{fSAD}}$ to CT-scored air trapping.

Figure S3: Pulmonary function evolution of a 36year-old female patient transplanted for nonspecific interstitial fibrosis with additional endobronchial stenting within 2 months after transplantation for collapse of the right bronchus. The $\mathrm{FEV}_{1}$ fluctuates during her later follow-up, declining below the BOS threshold ( $\geq 20 \mathrm{FEV}_{1}$ decline; red line) on several separate occasions. Despite this fluctuating pulmonary function, the patient remains BOS free at the end of follow-up. Two pulmonary function decreases could be explained by overt infection, which are indicated in the graph. The PRM measurements of the acquired CTs during her follow-up show a relatively stable percentage of PRM ${ }^{\mathrm{fSAD}}$ and PRM ${ }^{\text {Normal }}$ 